during a fairly short period of time, a few years at the most, as the arthropod cuticle does not withstand prolonged weathering.

The species found in the lower levels suggest the presence of rotting vegetation; but at the top large numbers of puparia of Lucilia and Calliphora (the bluebottle and greenbottle flies) indicated the presence of carrion and dung. It is interesting to note that the layer of clay which sealed the whole deposit was probably dumped on a day in late summer. The assemblage of species trapped immediately under it would suggest this, and also, although most of the fly puparia were vacated, a few clusters still contained remains of the immature flies.

For the identification of the specimens I am indebted to Mr. J. Balfour-Browne, and Dr. Owen Evans of the British Museum (Natural History), and Prof. F. E. Zeuner of the Institute of Archæology, University of London. I must also thank Mr. John Lunn of the Verulamium Museum for making the material available to me.

69 Manor Road,

Barnet, Herts.

1 Dobson, R. M., and Satchell, J. E., Nature, 177, 796 (1956).

\section{A Case of Limitation of Phenotypic Variation}

$J_{I N K S^{1}}$ suggests that where phenotypic variation is caused by a cytoplasmic system, the range of phenotypic expression attainable by the system would have quite definite limits imposed by the system itself (cytoplasmic limits) or, by the nonviability of phenotypes lying outside these limits (physiological limits). Cytoplasmic limits would result where further changes in cytoplasmic content are impossible, as when all cytoplasmic elements involved in the change are lost. Physiological limits would result where although more extreme changes in the cytoplasm are possible they would lead only to non-viable phenotypes. Jinks's results suggested a physiological basis to limits of variation obtained in Aspergillus glaucus.

Results from Neurospora crassa provide an example of a physiological limit to what appears to be cytoplasmically determined variation in the expression of a certain genotype. This genotype, which differs from the wild type by at least two genes, can show two different extremes of expression. At one extreme the culture forms no protoperithecia and instead produces many discrete areas of dark brown pigment (bk phenotype), while at the other extreme there is no sign of the dark pigment and weakly developed protoperithecia, which may be functional, are produced in limited parts of the culture ( $S$ phenotype). Stages intermediate between these two are formed. Cultures initiated from ascospores can show any stage in the range of phenotypes; but subcultures derived from them almost invariably show intermediate or $S$ phenotypes only. The $b k$ phenotype can be induced to appear, or reappear in subcultures by certain treatment, when its appearance, and later disappearance after the treatment are typical of the behaviour of a dauermodification. This and other evidence suggest that the phenotypic range results from the interaction of the genotype with varying cytoplasmic states or conditions. Prolonged continuation of the treatment used for inducing the appearance of phenotype $b k$ intensified formation of the brown pigment and was accompanied by a reduced growth rate and decreased viability. It appears that the induced cytoplasmic state led to the production of an abnormal phenotype, and that increase of the cytoplasmic state was limited by its causing an increasingly abnormal phenotype that led to the death of the cultures. In other words, there was a physiological limit to the extent to which the cytoplasmic state could be produced or selected for.

This view is supported by the results of a number of crosses involving these mutant strains. The crosses were first made after both strains had been subjected to the treatment leading to induction of phenotype $b k$ and again seven months later with the same strains, but from stocks which had never been subjected to the induction treatment. At both times crossing was carried out by simultaneous inoculations of parent strains in the crossing tubes. There was a marked difference in the results obtained from the two sets of crosses, and these are illustrated in Table 1 with data from a typical cross.

Table 1. Ascus Types Produced By Cross 3-1 A $\times$ 5-7a

\begin{tabular}{|c|c|c|c|c|c|c|c|}
\hline \multirow{3}{*}{ First crossing } & \multicolumn{6}{|c|}{ Ascus types } & \multirow{2}{*}{ No. of asci } \\
\hline & $\begin{array}{ll}L & L \\
L & L \\
L & L \\
L & L\end{array}$ & $\begin{array}{ll}L & L \\
L & L \\
L & L \\
L & L\end{array}$ & $\frac{b k}{b k}$ & $\begin{array}{l}b k \\
b k \\
b k \\
\end{array}$ & 二 & $\begin{array}{l}b k \\
b k \\
-\end{array}$ & \\
\hline & & & & & & & 9 \\
\hline \multirow[t]{2}{*}{ Second crossing } & \multirow[t]{2}{*}{$\begin{array}{ll}L & L \\
L & L \\
L & L\end{array}$} & \multirow[t]{2}{*}{$\begin{array}{ll}L & L \\
L & L \\
L & L\end{array}$} & \multirow[t]{2}{*}{$\begin{array}{l}S \\
S \\
S\end{array}$} & \multirow[t]{2}{*}{$\begin{array}{l}S \\
S \\
S\end{array}$} & \multirow[t]{2}{*}{$\begin{array}{c}S \\
\stackrel{S}{ } \\
b k\end{array}$} & \multirow[t]{2}{*}{$\begin{array}{l}S \\
b k \\
b k\end{array}$} & $\begin{array}{l}6 \\
3 \\
1\end{array}$ \\
\hline & & & & & & & 10 \\
\hline
\end{tabular}

$h$. Wild type protoperithecia ; $b k, b k$ phenotype; $S$, intermediate and' $S$ phenotypes; - , non-viable ascospore.

The cross was such that a $1: 1$ segregation of wild type to mutant would be expected. The mutant segregants in the progeny from the first crossing all had a strongly expressed $b k$ phenotype, and 50 per cent of the mutant ascospores were non-viable. In the progeny from the second crossing 87.5 per cent of the mutant segregants showed an intermediate or $S$ phenotype, while the remaining 12.5 per cent showed phenotype $b k$. There were no non-viable ascospores.

In all the crosses strong expression of phenotype $b k$ was associated with high ascospore non-viability, while expression of phenotype $S$ was associated with low ascospore non-viability. It appears that in the first series of crosses the cytoplasmic state of the progeny was such as to produce the extreme $b k$ phenotype, and that in many of the ascospores this cytoplasmic state was intensified to beyond the limit of phenotypic viability. In the progeny of the second series of crosses a more normal cytoplasmic state existed and led to a considerable amount of protoperithecial formation in the mutant segregates, and complete ascospore viability.

This work was carried out largely at the Department of Genetics, University of Adelaide, while I held a New Zealand National Research Fellowship.

\section{P. H. Fitzgerald}

Crop Research Division,

Department of Scientific and Industrial Research, Private Bag,

Christchurch, Now Zealand.

${ }^{1}$ Jinks, J. L., Proc. Roy. Soc., B, 148, 527 (1957), 